\title{
Cubic differentials and finite volume convex projective surfaces
}

\author{
YVES BENOIST \\ DOMINIQUE HULIN
}

\begin{abstract}
We prove that there exists a natural bijection between the set of finite volume oriented convex projective surfaces with nonabelian fundamental group and the set of finite volume hyperbolic Riemann surfaces endowed with a holomorphic cubic differential with poles of order at most 2 at the cusps.
\end{abstract}

30F30, 35J96, 53A15, 57M50, 53C56

\section{Introduction}

A projective structure $\mathcal{A}$ on an $n$-dimensional manifold $M$ is the data of a maximal atlas with values in the real projective space $\mathbb{R} \mathrm{P}^{n}$ and with transition functions in the group $\mathrm{PGl}_{n+1} \mathbb{R}$ of projective transformations. The charts glue together to define the developing map dev: $\widetilde{M} \rightarrow \mathbb{R} \mathrm{P}^{n}$, where $\widetilde{M}$ is the universal cover of $M$. The developing map is well-defined up to $\mathrm{PGl}_{n+1} \mathbb{R}$ and satisfies, for any loop $c$ in the fundamental group $\pi_{1}(M)$,

$$
\operatorname{dev} \circ c=\operatorname{hol}(c) \circ \operatorname{dev},
$$

where hol: $\pi_{1}(M) \rightarrow \mathrm{PGl}_{n+1} \mathbb{R}$ is the holonomy representation.

The projective structure is said to be properly convex when the developing map is a diffeomorphism onto a properly convex domain $\Omega \subset \mathbb{R} \mathrm{P}^{n}$, namely a domain that reads as a bounded convex open set in a suitable affine chart. The Hilbert distance on the properly convex subset $\Omega \subset \mathbb{R} \mathrm{P}^{n}$ is defined by

$$
d_{\Omega}(x, y)=|\log [x, y, a, b]|,
$$

where $[x, y, a, b]$ denotes the cross ratio of the quadruple $(x, y, a, b)$, and $a, b$ are the points of intersection of the line $(x y)$ and the boundary $\partial \Omega$ of $\Omega$. The Hilbert distance derives from a Finsler metric, which gives rise to a measure on $\Omega$ (Section 2.2). This measure, being invariant under the holonomy group hol $\left(\pi_{1}(M)\right)$, induces a measure $\mu_{F}$ on $M$. 
In this paper, we will mainly focus on projective surfaces $S$ with finite Finsler volume, namely such that $\mu_{F}(S)<\infty$. When $S$ is an oriented compact surface with negative Euler characteristic, François Labourie [10] and John Loftin [14] (see also C P Wang [22]) have parameterized the set of properly convex projective structures on $S$ by the data of a complex structure $J$ on $S$ together with a holomorphic cubic differential $U$ on $(S, J)$. The aim of this paper is to extend this result to noncompact projective surfaces with finite Finsler volume, hence positively answering the conjecture by Loftin in [13].

To an oriented surface $S$ equipped with a properly convex projective structure $\mathcal{A}$, we associate the following geometric objects. Denote by dev: $\widetilde{S} \rightarrow \Omega$ the developing map. Cheng-Yau's solution of the Monge-Ampère equation on $\Omega$ produces an imbedding $\widetilde{S} \hookrightarrow \mathbb{R}^{3}$ as an affine sphere (Theorem 2.5). Let $h$ be the induced "affine" metric on $S$ (Definition 2.2), $J$ be the conformal class of $h$-which is a complex structure on $S$-and $U$ be the Pick form (Remark-Definition 4.9) which is a holomorphic cubic differential on $(S, J)$.

Theorem 1.1 Let $S$ be an oriented surface with nonabelian fundamental group. The map $\mathcal{A} \rightarrow(J, U)$ is a bijection between

(1) the set of properly convex projective structures $\mathcal{A}$ on $S$ with finite Finsler volume,

(2) the set of hyperbolic Riemann surface structures $J$ on $S$ with finite volume together with a holomorphic cubic differential $U$ on $(S, J)$ with poles of order at most 2 at the cusps.

Remark 1.2 - Theorem 1.1 was conjectured by Loftin in [13], under the condition that $S$ has finite affine volume, instead of finite Finsler volume. In Proposition 2.6, we will prove these conditions to be equivalent by using Benzécri's cocompactness theorem (Theorem 2.7).

- Marquis proved in [18] that, when $\pi_{1}(S)$ is not finitely generated, $S$ does not admit any properly convex projective structure with finite Finsler volume.

- This bijection is a homeomorphism for the natural topologies on the two sets. Indeed, our construction ensures that the map $\mathcal{A} \rightarrow(J, U)$ is a continuous bijective map between two sets which are homeomorphic to a real vector space (see Marquis [17]).

Let us review the main ingredients of the proof. In order to prove that the map $\mathcal{A} \rightarrow(J, U)$ is well-defined we must first show that, when $(S, \mathcal{A})$ is assumed to have finite Finsler volume, the corresponding hyperbolic Riemann surface $(S, J)$ also has 
finite volume. Then we must control the Pick form at the cusps. To this end we first remark that, since the affine metric $h$ is complete (Corollary 3.5) and negatively pinched at infinity (Proposition 3.1), the generalized maximum principle of Yau ensures that $h$ is conformally quasi-isometric to the hyperbolic metric $h_{0}$ on $(S, J)$ (Proposition 5.1). Hence $\left(S, h_{0}\right)$ has finite volume, thus the ends of $(S, J)$ are parabolic (Corollary 5.4). The cocompactness theorem of Benzécri is then used again to provide estimates for the Pick form $U$, ensuring that the measure $|U|^{2 / 3}$ on $S$ has finite mass: this is the reason why $U$ will have poles of order no more than 2 at the cusps (Corollary 5.8).

The paper is organized as follows. In Section 2, we mainly recall standard material from affine geometry and Monge-Ampère equations, and prove the equivalence of the finite Finsler volume condition and the finite affine volume condition. In Section 3 we assume that $S$ is noncompact, and study the curvature of the affine metric at infinity. The Pick form of an affine sphere in $\mathbb{R}^{3}$ is defined in Section 4 , and the estimates for the Pick form that ensure that the map $\mathcal{A} \rightarrow(J, U)$ in Theorem 1.1 is well-defined are given in Section 5. In Section 6, we prove that this map is a bijection.

A right inverse map $(J, U) \rightarrow \mathcal{A}$ as in Theorem 1.1 was constructed by Loftin in [13]. This construction was the motivation for Loftin to state his conjecture. See also [15], where Loftin extends this construction to allow cubic differentials $U$ with poles of order at most 3.

Acknowledgements This work was completed while both authors were visiting the MSRI in Berkeley. We thank this institution for its support and hospitality. We also thank Alessio Figalli and Ludovic Marquis for interesting discussions on this topic.

\section{Affine spheres and the real Monge-Ampère equation}

In this section, we recall the definition of an affine sphere (references are for example Loftin [16] or Nomizu and Sasaki [19]) and the fundamental existence and uniqueness theorem by Cheng and Yau. We then derive from Benzécri's cocompactness theorem the equivalence of the finite Finsler volume condition and of the finite affine volume condition (Proposition 2.6).

\subsection{Affine spheres}

Let $M \subset \mathbb{R}^{n+1}$ be a hypersurface and $E=M \times \mathbb{R}^{n+1}$ be the trivial vector bundle of rank $n+1$ over $M$. The standard affine (flat) connection on $\mathbb{R}^{n+1}$ induces a flat connection $\nabla$ on $E$. Each choice of a transverse vector field $\xi: M \rightarrow \mathbb{R}^{n+1}$ yields 
a decomposition $E=T M \oplus L$, where $L$ stands for the trivial line bundle over $M$ spanned by $\xi$. It also yields a decomposition

$$
\begin{gathered}
\nabla_{X} Y=D_{X} Y+h(X, Y) \xi \in T M \oplus L, \\
\nabla_{X} \xi=-S(X)+\tau(X) \xi \in T M \oplus L,
\end{gathered}
$$

where $X$ and $Y$ are tangent vector fields. Observe that $D$ is a torsion-free connection on $T M, h$ is a symmetric 2 -form on $T M, S$ is an endomorphism of $T M$ and $\tau$ is a 1-form on $T M$.

Recall that a hypersurface $M \subset \mathbb{R}^{n+1}$ is locally strictly convex when it can locally be written in an affine coordinate system as the graph of a function with positive definite hessian.

Proposition 2.1 Assume that the hypersurface $M \subset \mathbb{R}^{n+1}$ is locally strictly convex. Then, there exists a unique transverse vector field $\xi$ for which

(1) $\tau=0$;

(2) $h$ is positive definite;

(3) $\left|\operatorname{det}\left(Y_{1}, \ldots, Y_{n}, \xi\right)\right|=1$ for any $h$-orthonormal frame $\left(Y_{1}, \ldots, Y_{n}\right)$ of $T M$.

Definition 2.2 This vector field $\xi$ is the "affine normal" of $M$. The corresponding connection $D$ on $T M$ is the "Blaschke connection", the metric $h$ is the "affine metric" on $M$ and the endomorphism $S: T M \rightarrow T M$ is the "affine shape operator". They are invariant under the action of the group $\mathrm{Sl}_{n+1}^{ \pm} \mathbb{R}$ of real matrices with determinant \pm 1 .

Proposition 2.1 will be a consequence of the following.

Lemma 2.3 Let $\xi$ and $\xi^{\prime}$ be two transverse vector fields on $M$, respectively associated with $(h, S, \tau)$ and $\left(h^{\prime}, S^{\prime}, \tau^{\prime}\right)$.

- If $\xi^{\prime}=\alpha \xi$, where $\alpha$ is a nonvanishing function on $M$, then we have that $\tau^{\prime}(X)=\tau(X)+(X \cdot \alpha) / \alpha$ and $h^{\prime}=h / \alpha$.

- If $\xi^{\prime}=\xi+\eta$ with $\eta$ a tangential vector field on $M$, then $\tau^{\prime}(X)=\tau(X)+h(X, \eta)$ and $h^{\prime}=h$.

Proof Straightforward. 
Proof of Proposition 2.1 Since $M$ is assumed to be strictly locally convex, $h$ is either positive or negative definite, and Lemma 2.3 ensures that the conformal class of $h$ does not depend on the choice of $\xi$. Now, starting with any transverse vector field $\xi$, the affine normal will be $\alpha \xi+\eta$ where $\alpha$ is the unique nonvanishing function on $M$ such that $\alpha \xi$ satisfies the positivity and normalization conditions 2 and 3, and $\eta$ is the unique tangent vector field for which the 1 -form $\tau_{\alpha \xi}$ corresponding to $\alpha \xi$ reads as $\tau_{\alpha \xi}=-h(\cdot, \eta)$.

Definition 2.4 A locally strictly convex hypersurface $M \subset \mathbb{R}^{n+1}$ with affine normal $\xi$ is an affine sphere with affine curvature -1 when the affine shape operator $S$ satisfies $S=-\mathrm{Id}$. In this case, the point $O=m-\xi(m)$ does not depend on $m \in M:$ it is called the center of the affine sphere.

The following existence and uniqueness theorem was conjectured by Calabi in [3] and proved by Cheng and Yau in [4]; see also Gigena [6].

Theorem 2.5 (Cheng-Yau) Let $\mathcal{C} \subset \mathbb{R}^{n+1}$ be an open convex cone that contains no line. There exists a unique embedded affine sphere $\mathcal{H} \subset \mathbb{R}^{n+1}$ with center the origin and affine curvature -1 which is asymptotic to the boundary of $\mathcal{C}$.

In any chart where $\mathcal{C}$ reads (with respect to an adapted unimodular frame) as

$$
\mathcal{C}=\{t(1, x) \mid x \in \Omega, t>0\},
$$

where $\Omega \subset \mathbb{R}^{n}$ is a bounded open convex set, $\mathcal{H}$ is the radial graph

$$
\mathcal{H}=\left\{\frac{-1}{u(x)}(1, x) \mid x \in \Omega\right\}
$$

where $u:=u_{\Omega} \in C^{0}(\bar{\Omega}) \cap C^{\infty}(\Omega)$ is the unique convex solution of the real MongeAmpère equation

$$
\begin{aligned}
\operatorname{det} D^{2} u & =(-1 / u)^{n+2} \text { in } \Omega, \\
u_{\mid \partial \Omega} & =0 .
\end{aligned}
$$

Theorem 2.5 will allow us to systematically identify any properly convex open subset $\Omega \subset \mathbb{R} \mathrm{P}^{n}$ with the corresponding affine sphere $\mathcal{H}$ with affine curvature -1 . The affine metric on $\mathcal{H}$ induces a Riemannian metric on $\Omega$ : we still denote it by $h$, and call it the affine metric on $\Omega$. The corresponding measure $\mu_{h}$ is called the affine measure on $\Omega$. 


\subsection{From properly convex projective manifolds to affine spheres}

Assume now that $M$ is an $n$-dimensional manifold equipped with a properly convex projective structure. Any of the developing maps identifies its universal cover $\widetilde{M}$ with a properly convex open set $\Omega \subset \mathbb{R} \mathrm{P}^{n}$. Since $\Omega$ is invariant under the action of the group $\operatorname{hol}\left(\pi_{1}(M)\right) \subset \mathrm{PGl}_{n+1} \mathbb{R}$, the uniqueness part in Theorem 2.5 ensures that the affine metric on $\Omega$ goes to the quotient to give a metric $h$ on $M$, that we will also call the "affine metric" on $M$.

On the other hand, the Hilbert distance on $\Omega$ derives from the Finsler metric defined for any point $x \in \Omega$ and any vector $X \in T_{x} \Omega$ as

$$
\|X\|_{F, \Omega}=\left(\frac{1}{\|x-a\|}+\frac{1}{\|x-b\|}\right)\|X\|,
$$

where $a$ and $b$ are the points of intersection of $\partial \Omega$ with the line defined by $(x, X)$ and $\|\cdot\|$ is any Euclidean norm on an affine chart with $\bar{\Omega} \subset \mathbb{R}^{n}$. To this Finsler metric, we associate the Borel measure $\mu_{F}$ on $\Omega \subset \mathbb{R}^{n}$ with density $\left(1 / m\left(B_{F, \Omega}(x, 1)\right)\right)$ with respect to the Lebesgue measure $m$ on $\mathbb{R}^{n}$, where $B_{F, \Omega}(x, 1)=\left\{X \in T_{x} \Omega,\|X\|_{F, \Omega}<1\right\}$ is the unit ball at the point $x$ for the Finsler metric (see eg Marquis [18]). The Finsler metric and the corresponding measure $\mu_{F}$ also go to the quotient to $M$.

Observe that both the affine and Finsler metrics on $M$ do not depend on the choice of the developing map.

Proposition 2.6 Let $M$ be a properly convex projective manifold. Then $M$ has finite Finsler volume if and only if it has finite affine volume.

The rest of the section is devoted to the proof of this proposition. The proof will rely on the continuity of the map $(x, \Omega) \rightarrow u_{\Omega}(x)$ (Proposition 2.8) and on the following cocompactness result due to Benzécri in [2], which will be used again later in this paper to provide estimates for the Pick measure (see Lemma 5.7).

Theorem 2.7 (Benzécri) Let $\mathcal{E}$ be the set of pairs $(x, \Omega)$, where $\Omega \subset \mathbb{R} \mathrm{P}^{n}$ is a properly convex open subset of $\mathbb{R} \mathrm{P}^{n}$ and $x$ is a point in $\Omega$, equipped with the Hausdorff topology. The natural action of $\mathrm{PGl}_{n+1} \mathbb{R}$ on $\mathcal{E}$ is cocompact.

As a consequence, the ratio of any two continuous $\mathrm{PGl}_{n+1} \mathbb{R}$ invariant positive functions on $\mathcal{E}$ will be bounded.

Now let $\mathcal{F}$ denote the set of pairs $(x, \Omega)$, where $\Omega \subset \mathbb{R}^{n}$ is a bounded convex domain and $x$ is a point in $\Omega$. 
Proposition 2.8 The value $u_{\Omega}(x)$ of the solution of the Monge-Ampère equation (2-2) at point $x$ depends continuously on $(x, \Omega) \in \mathcal{F}$.

Proof Let $\left(x_{0}, \Omega_{0}\right) \in \mathcal{F}$. When the convex set $\Omega$ is close to $\Omega_{0}$ in the Hausdorff topology,

$$
(1-\varepsilon) \Omega_{0} \subset \Omega \subset(1+\varepsilon) \Omega_{0}
$$

holds for dilations with center $x_{0}$ and $\varepsilon>0$ small. The proposition follows readily from the continuity of $u_{\Omega_{0}}: \Omega_{0} \rightarrow \mathbb{R}$, the easy fact that

$$
u_{t} \Omega_{0}(t x)=t^{n /(n+1)} u_{\Omega_{0}}(x)
$$

holds for all $x \in \Omega_{0}$ and $t>0$, and the following lemma.

Lemma 2.9 Let $\Omega_{1} \subset \Omega_{2}$ be two proper convex open subsets of $\mathbb{R}^{n}$. Then $u_{\Omega_{2}} \leq u_{\Omega_{1}}$ on $\Omega_{1}$.

Proof of Lemma 2.9 This assertion is a consequence of the maximum principle. Observe first that, since the function $u_{\Omega_{2}}$ is convex, $u_{\Omega_{2}} \leq u_{\Omega_{1}}$ holds on $\partial \Omega_{1}$. Assuming that the function $u_{\Omega_{1}}-u_{\Omega_{2}}$ reaches a negative minimum at an interior point $y \in \Omega_{1}$, the hessian $D_{y}^{2}\left(u_{\Omega_{1}}-u_{\Omega_{2}}\right)$ is positive semidefinite, hence

$$
\left(-1 / u_{\Omega_{2}}\right)^{n+2}=\operatorname{det} D_{y}^{2}\left(u_{\Omega_{2}}\right) \leq \operatorname{det} D_{y}^{2}\left(u_{\Omega_{1}}\right)=\left(-1 / u_{\Omega_{1}}\right)^{n+2},
$$

thus $\left|u_{\Omega_{1}}(y)\right|^{n+2} \leq\left|u_{\Omega_{2}}(y)\right|^{n+2}$, a contradiction.

Proof of Proposition 2.6 Let $\mu_{F}$ and $\mu_{h}$ denote respectively the Finsler and affine measures on a proper convex open subset of $\Omega \subset \mathbb{R} \mathrm{P}^{n}$, and let $\lambda$ be their ratio. It suffices to prove that the function $\lambda: \mathcal{E} \rightarrow] 0, \infty[$ is bounded, as well as bounded away from zero. This follows immediately from Benzécri's Theorem (Theorem 2.7) since

- $\lambda$ is invariant under $\mathrm{PGl}_{n+1} \mathbb{R}$, as both volume elements are;

- $\lambda$ is continuous. Indeed, in a neighborhood of $\left(x_{0}, \Omega_{0}\right)$ in $\mathcal{E}$, we may work in an affine chart where $\Omega_{0}$ reads as a bounded set $\Omega_{0} \subset \mathbb{R}^{n}$. It follows immediately from the definition that the density of the Finsler measure with respect to the Lebesgue measure $d x$ on $\mathbb{R}^{n}$ depends continuously on $(x, \Omega)$. It remains to check that the density of the affine measure also depends continuously on $(x, \Omega)$ : this follows from Proposition 2.8 and the formula

$$
\mu_{h}=\left(-u_{\Omega}\right)^{-n-1} d x,
$$

that we now prove. Let $\mathcal{H}$ be the affine sphere with affine curvature -1 and center the origin which is asymptotic to the cone $\mathcal{C}$ generated by $\Omega$ (Theorem 2.5). Since the 
affine normal at a point $y \in \mathcal{H}$ is $\xi(y)=y$, the Lebesgue measure $d v$ of $\mathbb{R}^{n+1}$ reads on the cone, in coordinates $v=s y$ with $(s, y) \in] 0, \infty\left[\times \mathcal{H}\right.$, as $d v=s^{n} d s \mu_{h}$. On the other hand, in coordinates $v=t(1, x)$ with $(t, x) \in] 0, \infty\left[\times \Omega\right.$, one has $d v=t^{n} d t d x$. The fact that $s=-u_{\Omega}(x) t$ yields (2-3).

\section{Estimates for the affine metric}

The aim of this section is to prove the following.

Proposition 3.1 Let $S$ be a noncompact properly convex projective surface with finite volume. Then, the curvature of the affine metric on $S$ tends to a negative constant at infinity.

Proposition 3.1 will rely on a priori interior estimates which were used by Cheng and Yau to solve the Monge-Ampère equation (see [4]). We recall the by now classical estimates for the Hölder norms of the solutions. For $x \in \mathbb{R}^{n}$ and $r>0$, let $B(x, r)$ denote the Euclidean open ball with center $x$ and radius $r$.

Proposition 3.2 Let $r>1, \delta>0$ and $0<c_{0} \leq c_{1}$.

Assume $\Omega \subset \mathbb{R}^{n}$ is a convex open subset such that $B(0,1) \subset \Omega \subset B(0, r)$, and let $v \in C^{0}(\bar{\Omega}) \cap C^{\infty}(\Omega)$ be a solution of the Monge-Ampère equation

$$
\begin{aligned}
\operatorname{det} D^{2} v & =f \quad \text { in } \Omega, \\
v_{\mid \partial \Omega} & =0,
\end{aligned}
$$

where $f \in C^{\infty}(\bar{\Omega})$ satisfies $c_{0} \leq f \leq c_{1}$. Then, for any $k \in \mathbb{N}, k \neq 1$, and $\left.\alpha \in\right] 0,1[$, one has

$$
\|v\|_{C^{k+2, \alpha}\left(\Omega_{\delta}\right)} \leq C,
$$

where $\Omega_{\delta}=\{x \in \Omega \mid d(x, \partial \Omega) \geq \delta\}$ and $C$ is a constant depending only on $r, \delta, c_{0}$, $c_{1}, k, \alpha$ and $\|f\|_{C^{k, \alpha}(\Omega)}$.

For $k=0$ this bound is Caffarelli's estimate (see Trudinger and Wang [21, Theorem 3.2ii]). For $k \geq 2$, these bounds result from the uniform ellipticity of the Monge-Ampère operator due to Pogorelov, and from the Schauder's estimates (see [21, Theorem 3.1]).

Corollary 3.3 For any $k \in \mathbb{N}$, the $k$-jet $J_{k}\left(u_{\Omega}\right)(x)$ of the solution of the MongeAmpère equation (2-2) on $\Omega$ at point $x$ depends continuously on $(x, \Omega) \in \mathcal{F}$. 
Proof of Corollary 3.3 We may assume $k \geq 1$. Let $\left(x_{j}, \Omega_{j}\right)$ converge to $(x, \Omega)$ in $\mathcal{F}$, and $K \subset \Omega$ be a compact neighborhood of $x$. We may assume that $K \subset \Omega_{j}$ for every $j$. Proposition 2.8 ensures that $u_{\Omega_{j}}$ converges uniformly to $u_{\Omega}$ on $K$ and, by convexity of the $u_{\Omega_{j}}$ 's, one has a uniform bound for the first derivative of every $u_{\Omega_{j}}$ on $K$. When applied to the function $v:=u_{\Omega_{j}}+\varepsilon$ on the domain $\Omega_{j, \varepsilon}:=\left\{u_{\Omega_{j}}<-\varepsilon\right\}$, where $\varepsilon>0$ is small enough, Proposition 3.2 gives a uniform bound for the jet of order $k+1$ of every $u_{\Omega_{j}}$ on $K$. After extracting a subsequence, the Ascoli-Arzéla theorem ensures that $u_{\Omega_{j}}$, as well as its derivatives of order at most $k$, converges uniformly on $K$.

As a first consequence, we obtain a uniform control of the affine metric in terms of the Finsler metric.

Proposition 3.4 There exists a constant $c>0$ such that, for any $(x, \Omega) \in \mathcal{E}$ and $X \in T_{x} \Omega$, we have

$$
(1 / c)\|X\|_{F, \Omega} \leq\|X\|_{h, \Omega} \leq c\|X\|_{F, \Omega} .
$$

Proof One easily verifies that the affine metric on $T_{x} \Omega$ can be expressed in terms of the 2 -jet of $u_{\Omega}$ at point $x$. The proposition follows again from Benzécri's Theorem (Theorem 2.7) since both metrics are $\mathrm{PGl}_{n+1} \mathbb{R}$ invariant and depend continuously on $(x, \Omega)$ : this is obvious for the Finsler metric from its definition, and is a consequence of Corollary 3.3 for the affine metric.

This observation allows us to recover the following, due to Calabi-Nirenberg; see Cheng and Yau [5, Corollary 2].

Corollary 3.5 The affine metric on any properly convex domain $\Omega \subset \mathbb{R} \mathrm{P}^{n}$ is complete.

Proof This is a consequence of Proposition 3.4, and of the completeness of the Finsler metric on $\Omega$.

From now on, we specialize to surfaces. Before going into the proof of Proposition 3.1, we state a preliminary result. A projective disk is defined as the orientable component of the complement of a proper conic in $\mathbb{R} \mathrm{P}^{2}$. In a suitable affine chart, it reads as the interior of an ellipse.

Proposition 3.6 Let $\Gamma \subset \mathrm{PGl}_{3} \mathbb{R}$ be a discrete subgroup preserving a properly convex domain $\Omega$ of $\mathbb{R} \mathrm{P}^{2}$. 
(1) There exists a convex fundamental domain $K \subset \Omega$ for the action of $\Gamma$ on $\Omega$, such that the map $K \rightarrow \Gamma \backslash \Omega$ is proper.

Assume moreover that the quotient $S=\Gamma \backslash \Omega$ has finite volume.

(2) The closure $\bar{K}$ of $K$ in $\mathbb{R} \mathrm{P}^{2}$ is a finite-sided polyhedron. Let $p$ be a point in $\bar{K} \cap \partial \Omega$. It corresponds to a cusp of $S$. The holonomy $\gamma$ of the cusp is regular unipotent, namely is generated by a matrix in the conjugacy class of

$$
\left(\begin{array}{lll}
1 & 1 & 0 \\
0 & 1 & 1 \\
0 & 0 & 1
\end{array}\right) .
$$

(3) There exist two projective disks $D_{1}$ and $D_{2}$ with

$$
D_{1} \subset \Omega \subset D_{2},
$$

and such that $\partial D_{1}$ and $\partial D_{2}$ osculate each other at the point $p \in \bar{K} \cap \partial \Omega$, namely the disks have a contact of order at least 2 at this point.

Remark 3.7 As a consequence, the curve $\partial \Omega$ not only admits a tangent at point $p$, but also osculating conics.

Assertion (1) is due to Lee [11], and assertion (2) to Marquis [18].
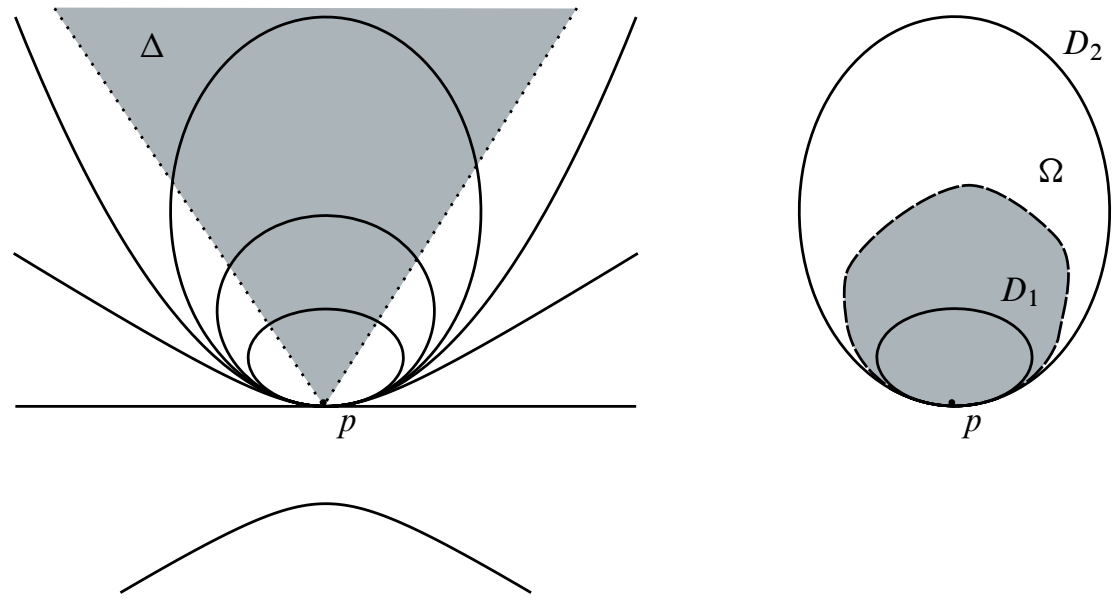

Figure 1: The pencil of conics-The disks $D_{1}$ and $D_{2}$ 
Proof We only have to prove assertion (3). We develop a strategy initiated by Benzécri in [2] (see Goldman [8, Proposition 6.14] and Marquis [18, Proposition 5.21]). We choose homogeneous coordinates $[x, y, z]$ with $p=[1,0,0]$, and where the holonomy of the cusp is generated by

$$
\gamma=\left(\begin{array}{lll}
1 & 1 & 0 \\
0 & 1 & 1 \\
0 & 0 & 1
\end{array}\right) .
$$

The holonomy preserves each quadratic form in the pencil that is generated by $z^{2}$ and $y^{2}-(y+2 x) z$. The corresponding pencil of conics has base point $p$ and reads in the chart $x=1$ (see Figure 1a) as

$$
\begin{aligned}
z^{2} & =0, \\
(y-z / 2)^{2}+(\mu-1 / 4) z^{2}-2 z & =0 \quad(\mu \in \mathbb{R}) .
\end{aligned}
$$

All these conics admit the line $z=0$ as a tangent at the point $p$ and, except for the singular one, they do osculate each other at this point.

Now pick an affine chart containing $\Omega$ as a bounded subset and new affine coordinates $(y, z)$ on this chart for which $p=(0,0)$ and the degenerate conic of the pencil still reads as $z^{2}=0$. In this chart the bounded domain $\Omega$, being invariant under $\gamma$, lies in the half-plane (say $z>0$ ) containing the ellipses in the pencil.

- We first build the exterior disk $D_{2}$. Choose $m \in \Omega$ and let $\Delta$ be the sector in the half-plane $\{z>0\}$ delimited by the line $(p m)$ and its image $\gamma(p m)=p \gamma(m)$. One of the conics in the pencil (actually, one of the hyperbolas) contains $\Delta$ in its interior $D_{2}$. This obviously gives $\Omega \cap \Delta \subset D_{2}$ hence, by invariance under $\gamma$,

$$
\gamma^{k}(\Omega \cap \Delta)=\Omega \cap \gamma^{k}(\Delta) \subset D_{2}, \quad \forall k \in \mathbb{Z},
$$

and finally $\Omega \subset D_{2}$.

- We now construct the interior disk. The domain $\Omega$, being convex, contains the triangle with vertices $p, m, \gamma(m)$. Since the ellipses in the pencil become tiny when the parameter $\mu$ goes to infinity, there exists one of them, say $D_{1}$, for which $D_{1} \cap \Delta$ is included is this triangle. We conclude as above that the disk $D_{1}$ satisfies $D_{1} \subset \Omega$.

Observe that the affine metric on a projective disk has constant negative curvature: this follows from the fact that $\mathrm{SO}(2,1) \subset \mathrm{Sl}_{3} \mathbb{R}$ acts transitively on each sheet of the hyperboloid. This observation will be crucial in the following. 
Proof of Proposition 3.1 We use the notation and conclusion of the previous proposition. Let us focus on the cusp $p \in \bar{K} \cap \Omega$. Choose a second point $q \neq p$ on the boundary of the exterior disk $D_{2}$. Let $r$ be the point of intersection of the tangent lines to $D_{2}$ at points $p$ and $q$, and $\left(\psi_{t}\right)_{t \in \mathbb{R}}$ be the 1-parameter group of projective transformations that reads as

$$
\psi_{t}=\left(\begin{array}{ccc}
e^{-t} & 0 & 0 \\
0 & e^{t} & 0 \\
0 & 0 & 1
\end{array}\right),
$$

with respect to the frame $(p, q, r)$.
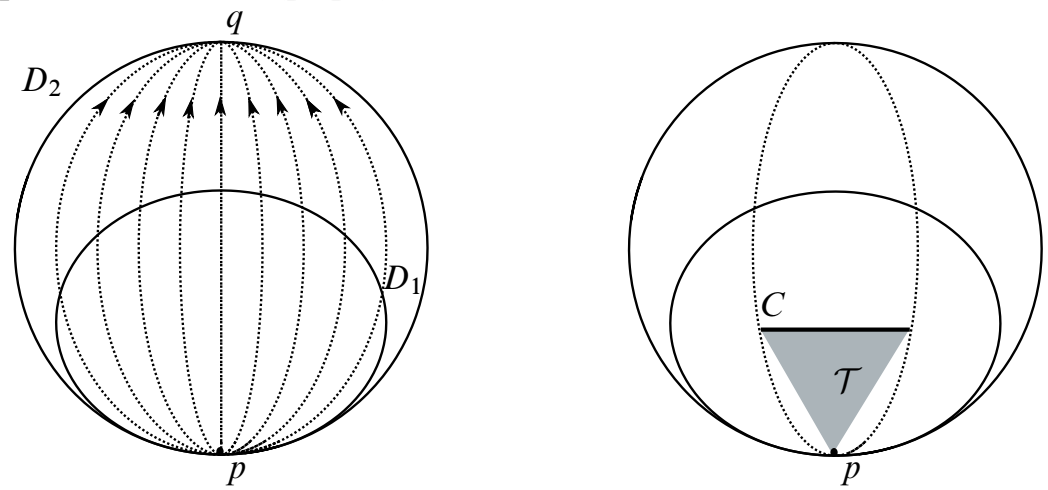

Figure 2: The orbits of $\left(\psi_{t}\right)$

In the corresponding homogeneous coordinates $[x, y, z]$, this 1 -parameter group preserves both quadratic forms $z^{2}$ and $x y$. Hence, each orbit of $\left(\psi_{t}\right)$ is an arc delimited by $p$ and $q$ either on a conic through $p$ and $q$ and with tangent $(p r)$ at $p$ and $(q r)$ at $q$, or on the line $(p q)$. Observe that, except for $\partial D_{2}$ itself, none of these conics osculate $\partial D_{2}$ at the point $p$ (Figure 2a).

Since $\partial D_{1}$ does osculate $\partial D_{2}$ at $p$, this implies that the orbit of any point $m$ in $D_{2}$ meets $D_{1}$ or, more specifically, that

$$
\forall m \in D_{2}, \exists T \in \mathbb{R}, \forall t \leq T, \psi_{t}(m) \in D_{1} .
$$

We infer that $\psi_{t}\left(D_{1}\right)$ converges to $D_{2}$ in the Hausdorff topology when $t$ goes to $+\infty$. Since $D_{1} \subset \Omega \subset D_{2}, \psi_{t}(\Omega)$ also converges to $D_{2}$. Thus, by Corollary 3.3, the curvature of the affine metric $h_{\psi_{t}(\Omega)}$ converges uniformly to the curvature of $h_{D_{2}}$, that is to a negative constant, on compact subsets of $D_{2}$ when $t \rightarrow+\infty$.

Let $\mathcal{T}$ be a triangular neighborhood of $p$ in $\bar{K}$ with interior $\stackrel{\circ}{\mathcal{T}} \subset D_{1}$. There exists a compact subset $C \subset D_{2}$ such that each orbit $\left(\psi_{t}(m)\right)_{t \in \mathbb{R}}$ of a point $m \in \mathcal{T} \cap \Omega$ intersects $C$ (Figure 2b). The farther $m \in \mathcal{T}$ is in the cusp (that is, the closer to $p$ ), the 
greater the values of $t$ for which $\psi_{t}(m) \in C$. Since the curvature of $h_{\Omega}$ at the point $m$ equals the curvature of $h_{\psi_{t}}(\Omega)$ at the point $\psi_{t}(\mathrm{~m})$, this concludes the proof.

\section{The Pick tensor}

The aim of this section, which basically follows the presentation by Labourie in [10], is to define the Pick form $U$ of an affine 2-dimensional sphere (Remark-Definition 4.9)which is the second component of the map $\mathcal{A} \rightarrow(J, U)$ in Theorem 1.1.

\subsection{From affine spheres to properly convex projective manifolds}

To an $n$-dimensional properly convex projective manifold $M$ with developing map dev: $\widetilde{M} \rightarrow \Omega \subset \mathbb{R} \mathrm{P}^{n}$, Theorem 2.5 associates an embedding of the universal cover $\widetilde{M} \hookrightarrow \mathbb{R}^{n+1}$ as the affine sphere with affine curvature -1 asymptotic to the boundary of the cone over $\Omega$. Moreover, the affine metric on $\widetilde{M}$ is complete (Corollary 3.5).

On the other hand, all complete affine spheres of $\mathbb{R}^{n+1}$ have been described by Cheng and Yau and An-Min Li in [5; 12], respectively, as follows, thus complementing Theorem 2.5 and proving the whole Calabi's conjecture stated in [3].

Theorem 4.1 (Cheng and Yau, An-Min Li) Let $\mathcal{H} \subset \mathbb{R}^{n+1}$ be an immersed hypersurface, which is an affine sphere with constant curvature -1 and center the origin. Assume that the corresponding affine metric on $\mathcal{H}$ is complete. Then $\mathcal{H}$ is embedded, and is asymptotic to the boundary of a convex cone $\mathcal{C} \subset \mathbb{R}^{n+1}$ which contains no line.

Thus, the data of a properly convex projective structure on the manifold $M$ is equivalent to the data of an immersion $\widetilde{M} \hookrightarrow \mathbb{R}^{n+1}$ of its universal cover as a complete affine sphere with affine curvature -1 and center the origin, which is equivariant under a representation $\pi_{1}(M) \rightarrow \mathrm{Sl}_{n+1}^{ \pm} \mathbb{R}$.

When $M \subset \mathbb{R}^{n+1}$ is an affine sphere with affine curvature -1 and $\xi$ is the affine normal, recall that the affine flat connection $\nabla$ on $\mathbb{R}^{n+1}$ induces a torsion-free connection $D$ on $T M$ as well as a positive definite 2-form $h$ on $T M$ which satisfies Equation (4-1) below. Indeed, in this case, Equation (2-1) holds with $S=-\mathrm{Id}$ and $\tau=0$ (see Proposition 2.1(1) and Definition 2.4). In the following subsection, we will study in an intrinsic way such pairs $(D, h)$ on $M$, where $D$ is a torsion-free connection and $h$ is a Riemannian metric. 


\subsection{The intrinsic geometry of an affine sphere}

Let $M$ be an $n$-dimensional manifold. The letters $X, Y, Z, W$ will denote tangent vector fields on $M$.

Let $D$ be a torsion-free connection on $T M, h$ be a positive definite symmetric 2 -form on $T M$ and $\xi$ be a nonvanishing section of the trivial line bundle $L$ over $M$. To these data, we associate a torsion-free connection $\nabla$ on $T M \oplus L$ by letting

$$
\begin{aligned}
\nabla_{X} Y & =D_{X} Y+h(X, Y) \xi, \\
\nabla_{X} \xi & =X .
\end{aligned}
$$

In the next three lemmas, we will explore the conditions under which the connection $\nabla$ will be flat and volume preserving.

Lemma 4.2 The curvature $R^{\nabla}$ of the connection $\nabla$ is given by

$$
\begin{aligned}
R^{\nabla}(X, Y) Z & =R(X, Y) Z-h(Y, Z) X+h(X, Z) Y+d^{D} h(X, Y, Z) \xi, \\
R^{\nabla}(X, Y) \xi & =0,
\end{aligned}
$$

where $R$ is the curvature of $D$ and $d^{D} h(X, Y, Z):=\left(D_{X} h\right)(Y, Z)-\left(D_{Y} h\right)(X, Z)$.

Proof Use the definition $R^{\nabla}(X, Y) Z:=\nabla_{X} \nabla_{Y} Z-\nabla_{Y} \nabla_{X} Z-\nabla_{[X, Y]} Z$.

Lemma 4.3 Write $D=D^{h}+A$, where $D^{h}$ is the Levi-Civita connection of the Riemannian metric $h$ and $A$ is a section of the bundle $T^{*} M \otimes$ End TM. Then

(1) $A(X) Y=A(Y) X$;

(2) the connection $\nabla$ defined by Equation (4-1) is flat if and only if

(a) each endomorphism $A(X)$ is symmetric with respect to $h$;

(b) $d^{D^{h}} A=0$, where $d^{D^{h}} A(X, Y):=\left(D_{X}^{h} A\right)(Y)-\left(D_{Y}^{h} A\right)(X)$;

(c) $R^{h}(X, Y) Z+[A(X), A(Y)] Z=h(X, Z) Y-h(Y, Z) X$, where $R^{h}$ is the curvature of $D^{h}$.

Proof (1) Both connections $D$ and $D^{h}$ are torsion-free.

(2) The normal part of $R^{\nabla}$ vanishes if and only if $d^{D} h=0$ (Lemma 4.2). Since $D^{h} h=0$,

$$
\left(D_{X} h\right)(Y, Z)=-h(A(X) Y, Z)-h(Y, A(X) Z)
$$

holds, thus (1) yields

$$
d^{D} h(X, Y, Z)=h(X, A(Z) Y)-h(Y, A(Z) X),
$$


which proves (a).

The tangential part of $R^{\nabla}$ vanishes if and only if $R(X, Y) Z=h(Y, Z) X-h(X, Z) Y$. A simple computation gives

$$
R(X, Y) Z=R^{h}(X, Y) Z+\left(d^{D^{h}} A(X, Y)\right) Z+[A(X), A(Y)] Z,
$$

and hence the result, since for fixed $X$ and $Y$, we have that the endomorphism $Z \rightarrow h(X, Z) Y-h(Y, Z) X$, as well as those defined by each term in the right-hand side of (4-3), are skew symmetric with respect to $h$, except for $Z \rightarrow\left(d^{D^{h}} A(X, Y)\right) Z$, which is symmetric.

Lemma 4.4 Assume that $M$ is oriented. Define a volume form $\omega$ on $T M \oplus L$ by imposing that $\omega\left(X_{1}, \ldots, X_{n}, \xi\right)=1$, whenever $\left(X_{1}, \ldots, X_{n}\right)$ is a positive $h$-orthonormal frame of TM. Then

$$
\nabla \omega=0 \quad \text { if and only if } \quad D \omega_{h}=0 \quad \text { if and only if } \forall X, \operatorname{Tr} A(X)=0,
$$

where $\omega_{h}$ denotes the volume form of $h$ and $\operatorname{Tr}$ is the trace operator.

Remark 4.5 This lemma is motivated by Proposition 2.1(3), where a constant volume form on $\mathbb{R}^{n+1}$ is used to normalize the affine normal vector.

Proof Observe that, since $\nabla_{X} Y-D_{X} Y$ is colinear to $\xi$ and $\nabla_{X} \xi$ is a tangent vector field, one has

$$
\begin{aligned}
D_{X} \omega_{h}\left(X_{1}, \ldots, X_{n}\right) & =X \cdot \omega\left(X_{1}, \ldots, X_{n}, \xi\right)-\sum_{i}^{n} \omega\left(X_{1}, \ldots, \nabla_{X} X_{i}, \ldots, X_{n}, \xi\right) \\
& =\nabla_{X} \omega\left(X_{1}, \ldots, X_{n}, \xi\right) .
\end{aligned}
$$

This proves the first equivalence. On the other hand, since $D=D^{h}+A$ and $D^{h} \omega_{h}=0$, one has

$$
\begin{aligned}
D_{X} \omega_{h}\left(X_{1}, \ldots, X_{n}\right) & =-\sum_{i=1}^{n} \omega_{h}\left(X_{1}, \ldots, A(X) X_{i}, \ldots, X_{n}\right) \\
& =-\operatorname{Tr} A(X) \omega_{h}\left(X_{1}, \ldots, X_{n}\right),
\end{aligned}
$$

which concludes the proof.

Corollary 4.6 Define a tensor $C$ on $T M$ by letting

$$
C(X, Y, Z):=h(A(X) Y, Z) .
$$

The 3-tensor $C$ is symmetric if and only if each endomorphism $A(X)$ is symmetric with respect to $h$. In that case, one also has $\left(D_{X} h\right)(Y, Z)=-2 C(X, Y, Z)$. 
Proof The first assertion immediately follows from Lemma 4.3(1), and the second from the fact that $D^{h} h=0$.

Remark-Definition 4.7 Assume that $M \subset \mathbb{R}^{n+1}$ is an affine sphere, and that $D$ and $h$ are respectively the Blaschke connection and the affine metric on $M$. Then the corresponding 3-tensor $C$ is symmetric. It is called the "Pick tensor" of $M$. The Pick tensor $C$ vanishes if and only if the affine sphere $M$ is part of a hyperquadric (see Cheng and Yau [5, Theorem 1]).

\subsection{The Pick form}

When specializing the previous section to surfaces, we have the following.

Lemma 4.8 Assume that the manifold $M$ is 2-dimensional and oriented. Let $J$ denote the underlying complex structure to $h$ and suppose that the 3-tensor $C$ is symmetric. Then, one has the equivalence: each endomorphism $A(X)$ is trace-free and $d^{D^{h}} A=0$ if and only if $C$ is the real part of a holomorphic cubic differential $U$ on $(M, J)$.

Proof Pick local isothermal coordinates $(x, y)$ such that the frame $\left(\partial_{x}, \partial_{y}\right)$ is positively oriented, and let $z=x+i y$. The affine metric reads as $h=e^{2 w}\left(d x^{2}+d y^{2}\right)$. Observe that both endomorphisms $A\left(\partial_{x}\right)$ and $A\left(\partial_{y}\right)$ are trace-free if and only if the symmetric tensor $C$ reads in this chart as

$$
C=P d x^{3}-P d x d y^{2}-Q d x^{2} d y+Q d y^{3},
$$

where $P$ and $Q$ are real-valued functions or, in other words, if

$$
C=\operatorname{Re}\left((P+i Q)(d x+i d y)^{3}\right)=\operatorname{Re}\left(f(z) d z^{3}\right),
$$

where $f=P+i Q$ is complex-valued. Assume that these conditions are satisfied. It remains to check under which condition $d^{D^{h}} A=0$ holds. Observe first that this is equivalent to requiring that $d^{D^{h}} C=0$, where

$$
d^{D^{h}} C(X, Y, Z, W):=\left(D_{X}^{h} C\right)(Y, Z, W)-\left(D_{Y}^{h} C\right)(X, Z, W) .
$$

Let $\partial_{z}=(1 / 2)\left(\partial_{x}-i \partial_{y}\right)$ and $\partial_{\bar{z}}=(1 / 2)\left(\partial_{x}+i \partial_{y}\right)$. Since $h\left(\partial_{z}, \partial_{z}\right)=h\left(\partial_{\bar{z}}, \partial_{\bar{z}}\right)=0$ while $h\left(\partial_{z}, \partial_{\bar{z}}\right)=(1 / 2) e^{2 w}$, and since the connection $D^{h}$ is torsion-free, we infer that $D_{\partial z}^{h} \partial z$ and $D_{\partial_{\bar{z}}}^{h} \partial_{\bar{z}}$ are respectively colinear to $\partial_{z}$ and $\partial_{\bar{z}}$, and $D_{\partial_{\bar{z}}}^{h} \partial_{z}=D_{\partial_{z}}^{h} \partial_{\bar{z}}=0$.

The definition of $C$ ensures that, for any vector field $Y$, one has $C\left(Y, \partial_{z}, \partial_{\bar{z}}\right)=0$. It then follows from the expression of the Levi-Civita connection $D^{h}$ with respect to the 
frame $\left(\partial_{\bar{z}}, \partial_{\bar{z}}\right)$ that $\left(D_{X}^{h} C\right)\left(Y, \partial_{z}, \partial_{\bar{z}}\right)=0$ holds for any vector fields $X$ and $Y$. Since the tensor $d^{D^{h}} C$ is skew-symmetric with respect to the first couple of variables, and symmetric with respect to the second, it follows that $d^{D^{h}} C=0$ if and only if

$$
d^{D^{h}} C\left(\partial_{\bar{z}}, \partial_{z}, \partial_{z}, \partial_{z}\right)=0 .
$$

The easy fact that $d^{D^{h}} C\left(\partial_{\bar{z}}, \partial_{z}, \partial_{z}, \partial_{z}\right)=\partial f / \partial \bar{z}$ now ends the proof.

Remark-Definition 4.9 It follows from Lemma 4.8 that, when $\mathcal{H} \subset \mathbb{R}^{3}$ is a 2dimensional affine sphere, the Pick tensor $C$ reads as $C=\operatorname{Re} U$ where the "Pick form" $U$ is a holomorphic cubic differential on $(\mathcal{H}, J)$. We will also denote by $U$ the Pick form on any properly convex domain of $\mathbb{R} \mathrm{P}^{2}$, and on any properly convex projective surface.

\section{The conformal structure of $(S, h)$ and the Pick form}

Let $S$ be an oriented surface with nonabelian fundamental group, equipped with a finite volume properly convex projective structure. We first explore the conformal structure of the affine metric on $S$, and show that it has only parabolic ends (Corollary 5.4). Then, we prove the estimates for the Pick form at the cusps (Corollary 5.8) that will ensure that the map $\mathcal{A} \rightarrow(J, U)$ of our main theorem (Theorem 1.1) is well-defined.

Proposition 5.1 Let $S$ be an oriented finite volume properly convex projective surface with nonabelian fundamental group, $h$ be the affine metric on $S$ and write $h=e^{2 v} h_{0}$ (with $v \in C^{\infty}(S)$ ) where $h_{0}$ is the hyperbolic metric in the conformal class of $h$. Then $h$ and $h_{0}$ are "conformally quasi-isometric", namely the conformal factor $v$ is bounded.

Proof Since $S$ has nonabelian fundamental group, it follows from the uniformization theorem that there exists a hyperbolic metric $h_{0}$ in the conformal class of $h$. The affine metric is complete (Corollary 3.5) and its curvature is negatively pinched at infinity (Proposition 3.1). This makes Proposition 5.1 a special case of the following version of the Ahlfors-Schwarz lemma; see Ahlfors [1].

Lemma 5.2 Let $h=e^{2 v} h_{0}$ be two conformal metrics on a noncompact surface $S$, and with respective curvatures $K$ and $K_{0}$. Assume that both metrics are complete and that

$$
-a \leq K, \quad K_{0} \leq-b<0,
$$

holds at infinity for some positive constants $a$ and $b$. Then $h$ and $h_{0}$ are conformally quasi-isometric. 
This lemma will be, as in Troyanov [20], a consequence of the generalized maximum principle of Yau [23].

Theorem 5.3 (Yau) Let $w$ be a smooth function on a complete Riemannian manifold $\left(M, h_{0}\right)$ whose Ricci curvature is bounded below. If $w$ is bounded above and does not reach its maximum, there exists a sequence of points $p_{n} \in M$ going to infinity, and such that $w\left(p_{n}\right) \rightarrow \sup _{M} w,\left|\nabla_{0} w\left(p_{n}\right)\right| \rightarrow 0$, and with $\lim \Delta_{0} w\left(p_{n}\right) \in[0, \infty]$.

Here $\nabla_{0}$ and $\Delta_{0}$ respectively denote the gradient and the Laplace operator with respect to the metric $h_{0}$, with the sign convention $\Delta_{0}:=-\operatorname{Tr} D^{h_{0}} \nabla_{0}$.

Proof of Lemma 5.2 By symmetry, it is sufficient to prove that $v$ is bounded above. If this is not the case, the function $w:=1 /\left(1+e^{-v}\right)$ does not reach its maximum, and there exists a sequence $p_{n} \in S$ as in Theorem 5.3, with $w\left(p_{n}\right) \rightarrow 1$. A simple computation yields

$$
\Delta_{0} w=\Delta_{0} v \frac{e^{-v}}{\left(1+e^{-v}\right)^{2}}-\left|\nabla_{0} w\right|^{2}\left(e^{v}-e^{-v}\right) .
$$

Plugging the equation for curvatures

$$
\Delta_{0} v=K e^{2 v}-K_{0}
$$

into this expression gives

$$
\Delta_{0} w=K \frac{e^{v}}{\left(1+e^{-v}\right)^{2}}-K_{0} \frac{e^{-v}}{\left(1+e^{-v}\right)^{2}}-\left|\nabla_{0} w\right|^{2}\left(e^{v}-e^{-v}\right),
$$

hence a contradiction when evaluated at the points $p_{n}$, since the first term goes to $-\infty$, the second one goes to zero and the third one is nonpositive when $n \rightarrow \infty$.

Corollary 5.4 Let $S$ be an oriented finite volume properly convex projective surface with nonabelian fundamental group. Let $J$ be the underlying complex structure to the affine metric $h$ on $S$. Then $S$ has finite topological type and the Riemann surface $(S, J)$ has only parabolic ends. In other words, there exist a compact Riemann surface $\bar{S}$ and a finite set $\left\{p_{1}, \ldots, p_{k}\right\} \subset \bar{S}$ such that $(S, J)$ identifies with $\bar{S} \backslash\left\{p_{1}, \ldots, p_{k}\right\}$.

The fact that $S$ has finite topological type is due to Marquis, and was used in the proof of Proposition 3.1.

Proof Since $h$ has finite volume, Proposition 5.1 ensures that the hyperbolic metric $h_{0}$ on $(S, J)$ also has finite volume, hence all its ends are parabolic. 
Remark 5.5 In a complex chart $\{z \in \mathbb{C}|0<| z \mid \leq 1 / 2\}$ around a puncture, the metrics $h$ and $h_{0}$ are conformally quasi-isometric to the Beltrami metric $g=|d z|^{2} /|z|^{2}|\log | z||^{2}$. This is again a consequence of Lemma 5.2.

To conclude this section, it only remains to prove that the Pick form $U$ on $S$ (RemarkDefinition 4.9) is a meromorphic form on the compactification $\bar{S}$ of $S$, with a pole of order at most 2 at each puncture $p$.

Definition 5.6 Let $\Omega$ be a properly convex open subset of $\mathbb{R} \mathrm{P}^{2}$ and $U$ be the corresponding Pick form on $\Omega$. We define the Pick measure $\mu_{P}:=|U|^{2 / 3}$ on $\Omega$ by

$$
\mu_{P}=|f(z)|^{2 / 3}|d z|^{2}
$$

in any complex chart where $U$ reads as $U(z)=f(z) d z^{3}$.

Lemma 5.7 There exists a constant $C>0$ such that, for any properly convex open subset $\Omega$ of $\mathbb{R P}^{2}$, the ratio $\Lambda \in\left[0, \infty\left[\right.\right.$ of the Pick measure $\mu_{P}$ by the affine measure $\mu_{h}$ is uniformly bounded above by $C$.

Proof Since the Pick measure can be expressed in terms of the 3 -jet of $u_{\Omega}$, then Corollary 3.3 and Equation (2-3) ensure that the ratio $\Lambda$ depends continuously on $(x, \Omega) \in \mathcal{E}$. This ratio being invariant under the action of $\mathrm{PGl}_{3} \mathbb{R}$, we conclude by Benzécri's cocompactness theorem (Theorem 2.7) that $\Lambda$ is bounded.

Corollary 5.8 Let $S$ be an oriented finite volume properly convex projective surface with nonabelian fundamental group. Then the Pick form $U$ is a meromorphic cubic differential on the compactification $\bar{S}$ of $(S, J)$, and with poles of order at most 2 at each puncture $p_{i}(1 \leq i \leq k)$.

Proof Work in a complex chart $D^{*}=\{z \in \mathbb{C}, 0<|z| \leq 1 / 2\}$ around a cusp of $(S, J)$ and let

$$
U(z)=f(z) d z^{3},
$$

where $f$ is a holomorphic function on the punctured disk $D^{*}$. Since the affine metric $h$ is conformally quasi-isometric to the Beltrami metric in the cusp (Remark 5.5), the boundedness of $\Lambda$ (Lemma 5.7) implies that there exists a constant $c>0$ such that

$$
|f(z)| \leq \frac{c}{|z|^{3}|\log | z||^{3}} \text { holds for } 0<|z| \leq 1 / 2 .
$$

Hence $f$ is meromorphic on the disk $D=\{|z| \leq 1 / 2\}$, with a pole of order at most 2 at the origin.

We just proved that the map $\mathcal{A} \rightarrow(J, U)$ is well-defined. This concludes the first part of the proof of Theorem 1.1. 


\section{Wang's equation}

In this final section, we wrap up the proof of our main theorem (Theorem 1.1) by constructing the inverse map $(J, U) \rightarrow \mathcal{A}$ as in Loftin's thesis [13].

\subsection{Retrieving the projective structure}

In this subsection, we continue the discussion in Section 4, and characterize those pairs $(D, h)$ of a torsion-free connection $D$ and a positive symmetric 2-form $h$ on the tangent bundle of a manifold $M$ which come from an immersion $M \hookrightarrow \mathbb{R}^{n+1}$ as an affine sphere.

Proposition 6.1 Let $M$ be an $n$-dimensional manifold endowed with a torsionfree connection $D$ and $h$ be a positive definite symmetric 2-form on $M$. Write $D=D^{h}+A$, where $D^{h}$ denotes the Levi-Civita connection of $h$ and $A$ is a section of $T^{*} M \otimes$ End $T M$.

Assume the manifold $M$ to be simply connected. Then $D$ is the Blaschke connection and $h$ is the affine metric corresponding to an immersion $M \hookrightarrow \mathbb{R}^{n+1}$ as an affine sphere with constant curvature -1 if and only if the following hold:

(a) each endomorphism $A(X)$ is trace-free and symmetric with respect to $h$;

(b) $d^{D^{h}} A=0$;

(c) $R^{h}(X, Y) Z+[A(X), A(Y)] Z=h(X, Z) Y-h(Y, Z) X$, where $R^{h}$ is the Riemannian curvature tensor of $h$.

Proof Lemma 4.3 and Lemma 4.4 ensure that conditions (a), (b) and (c) are necessary. Assume now that these conditions are satisfied and choose a nonvanishing section $\xi$ of the trivial line bundle over $M$. Then, the connection $\nabla$ on $T M \oplus L$ defined by (4-1) is flat, hence it identifies the bundle $T M \oplus L$ with the trivial bundle $\mathbb{R}^{n+1} \times M \rightarrow M$ and the section

$$
m \in M \rightarrow \xi(m) \in T_{m} M \oplus L_{m} \simeq \mathbb{R}^{n+1}
$$

provides an immersion of $M$ in $\mathbb{R}^{n+1}$ such that the connection $\nabla$ derives from the affine flat connection on $\mathbb{R}^{n+1}$. Moreover since each endomorphism $A(X)$ is supposed to be trace-free, the form $\omega$ in Lemma 4.4, being parallel with respect to $\nabla$, is the restriction to $M$ of a multiple of the constant volume form det on $\mathbb{R}^{n+1}$. Hence, after renormalizing the determinant, $\xi$ identifies with the affine normal of this immersion (Proposition 2.1). Equation (4-1) now tells us that the affine shape operator of this immersion is $S=-\mathrm{Id}$, that is, $M$ is immersed in $\mathbb{R}^{n+1}$ as an affine sphere with affine curvature -1 . 


\subsection{Stating Wang's equation}

We now specialize to surfaces and introduce Wang's equation, which relates the Pick form $U$ of an affine sphere (Remark-Definition 4.9) and the conformal factor between the affine metric $h$ and its uniformization $h_{0}$.

Let $S$ be an oriented surface with nonabelian fundamental group. The construction of the reciprocal map $(J, U) \rightarrow \mathcal{A}$ in Theorem 1.1 goes as follows. Given a pair $(J, U)$, where $J$ is a complex structure on $S$ and $U$ is a holomorphic cubic differential on $(S, J)$, we want to retrieve the properly convex projective structure $\mathcal{A}$. As explained in Section 4.1, this boils down to constructing a suitable embedding $\widetilde{S} \subset \mathbb{R}^{3}$ of the universal cover of $S$ as an affine sphere.

We first define a symmetric 3-tensor on $S$ by letting $C=\operatorname{Re} U$. Let $h_{0}$ be the hyperbolic metric on $(S, J)$. To each conformal metric $h=e^{2 v} h_{0}$ on $S$ we associate the section $A$ of the bundle $T^{*} S \otimes$ End $T S$ for which $C(X, Y, Z):=h(A(X) Y, Z)$ (see Corollary 4.6). We then define a torsion-free connection $D$ on $T S$ by $D:=D^{h}+A$, where $D^{h}$ denotes the Levi-Civita connection of $h$. These data $(D, h)$ lift to the universal cover $\widetilde{S}$, and Proposition 6.1 gives necessary and sufficient conditions for this pair to correspond to an immersion of $\widetilde{S}$ in $\mathbb{R}^{3}$ as an affine sphere.

Remark 6.2 When dealing with surfaces, we have proved in Lemma 4.8 that the above conditions (a) and (b) in Proposition 6.1 amount to saying that the tensor $C(X, Y, Z)=h(A(X) Y, Z)$ is the real part of a holomorphic cubic differential $U$ on $(S, J)$. Thus, it only remains to examine condition (c), which we will do in the next corollary.

Let $A_{0}$ be the section of $T^{*} S \otimes$ End TS associated to $C$ through the hyperbolic metric $h_{0}$, namely such that $C(X, Y, Z):=h_{0}\left(A_{0}(X) Y, Z\right)$, and let $\Delta_{0}:=-\operatorname{Tr} D^{h_{0}} \nabla_{0}$ denotes the Laplace operator on $\left(S, h_{0}\right)$.

Corollary 6.3 Let the function $k: S \rightarrow \mathbb{R}$ be defined as

$$
k:=-h_{0}\left(\left[A_{0}\left(X_{1}^{0}\right), A_{0}\left(X_{2}^{0}\right)\right] X_{2}^{0}, X_{1}^{0}\right),
$$

where $\left(X_{1}^{0}, X_{2}^{0}\right)$ is any orthonormal frame for the hyperbolic metric $h_{0}$.

The pair $(D, h)$ derives from an immersion of $\widetilde{S}$ in $\mathbb{R}^{3}$ as an affine sphere with affine curvature -1 if and only if the conformal factor $v$ is solution of Wang's equation

$$
\Delta_{0} v=-e^{2 v}+1+k e^{-4 v} .
$$


Proof Condition (c) is equivalent to

$$
h\left(R^{h}\left(X_{1}, X_{2}\right) X_{2}, X_{1}\right)+h\left(\left[A\left(X_{1}\right), A\left(X_{2}\right)\right] X_{2}, X_{1}\right)=-1,
$$

where $\left(X_{1}, X_{2}\right)$ is any orthonormal basis for the metric $h$. It is elementary to check that

$$
-h\left(\left[A\left(X_{1}\right), A\left(X_{2}\right)\right] X_{2}, X_{1}\right)=e^{-6 v} k .
$$

Taking into account the equation for curvature $\Delta_{0} v=K_{h} e^{2 v}+1$-where $K_{h}$ is the curvature of the affine metric $h$-reduces this condition to Wang's equation (6-2).

\subsection{Solving Wang's equation}

In this section, we finally end the proof of Theorem 1.1 by proving that Wang's equation admits a unique bounded solution.

Let $S$ be an oriented surface equipped with a pair $(J, U)$, where $J$ is a complex structure and $U$ is a holomorphic cubic differential as in Theorem 1.1. If these data derive (by the construction of Sections 2-4) from a properly convex structure on $S$ with finite volume, we have seen that the corresponding affine metric $h$ is complete and must read as $h=e^{2 v} h_{0}$, where $h_{0}$ is the hyperbolic metric on $(S, J)$ and the function $v$ is a bounded solution of Wang's equation (Proposition 5.1 and Corollary 6.3).

Lemma 6.4 Let $(S, J)$ be a hyperbolic Riemann surface with finite volume, and $U$ be a holomorphic cubic differential with poles of order at most 2 at the cusps. Then, the function $k$ defined by Equation (6-1) is nonnegative and bounded.

Proof It is elementary to check that $k$ reads as $k=2 e^{-6 \varphi}|f|^{2}$ in isothermal coordinates where $h_{0}=e^{2 \varphi}\left(d x^{2}+d y^{2}\right)$ and $U=f(z) d z^{3}$. The hypothesis on $U$ and Remark 5.5 ensure that $k$ is bounded on $S$.

Proposition 6.5 (Loftin [13]) Let $\left(S, h_{0}\right)$ be a Riemannian surface, and $k: S \rightarrow \mathbb{R}^{+}$ be a smooth non negative bounded function. Then, Wang's equation (6-2)

$$
\Delta_{0} v=-e^{2 v}+1+k e^{-4 v}
$$

admits bounded solutions, which are $C^{\infty}$. When $S$ is compact, or the metric $h_{0}$ is complete and with curvature bounded from below, such a bounded solution is unique.

End of proof of Theorem 1.1 Let $v$ be the unique bounded solution of Wang's equation (6-2). The conformal metric $h=e^{2 v} h_{0}$ on $S$ is complete. The affine metric $h$ and the Pick form $U$ lift to $\widetilde{S}$, and Corollary 6.3 and Theorem 4.1 ensure that these 
data correspond to an embedding $\widetilde{S} \hookrightarrow \mathbb{R}^{3}$ which is asymptotic to a convex cone $\mathcal{C} \subset \mathbb{R}^{3}$. Such a bounded solution being unique, this embedding is equivariant under a representation $\pi_{1}(S) \rightarrow \mathrm{Sl}_{3}^{ \pm} \mathbb{R}$, hence provides the desired properly convex projective structure on $S$.

For the sake of completeness we now give a proof of Proposition 6.5, which relies on the classical so-called method of upper and lower solutions.

Proof of Proposition 6.5 Define $f: S \times \mathbb{R} \rightarrow \mathbb{R}$ by $f(x, t):=-e^{2 t}+1+k(x) e^{-4 t}$. Since $k$ is positive and bounded, there exist constant functions $v_{-} \leq v_{+}$which provide respectively lower and upper solutions for Equation (6-2), namely such that $\Delta_{0} v_{-} \leq f\left(x, v_{-}\right)$and $\Delta_{0} v_{+} \geq f\left(x, v_{+}\right)$. Moreover, there exists a constant $c>0$ such that, for any $v_{-} \leq s<t \leq v_{+}$and $x \in S$,

$$
f(x, s)-c(t-s) \leq f(x, t)<f(x, s)
$$

holds.

Assume first that the surface $S$ is noncompact, and choose an exhaustion $\Sigma_{k} \subset \stackrel{\circ}{\Sigma}_{k+1}$, $\cup \Sigma_{k}=S$ of $S$ by compact surfaces with smooth boundaries.

Lemma 6.6 Let $\Sigma$ be a compact surface with smooth boundary. Let $\psi$, $v_{+}$be two functions in $C^{\infty}(\Sigma)$ and $c>0$ be a constant. Then, there exists a solution $w \in C^{\infty}(\Sigma)$ of the linear Dirichlet problem

$$
\begin{aligned}
\Delta_{0} w & =-c w+\psi \quad \text { on } \stackrel{\circ}{\Sigma}, \\
w & =v_{+} \quad \text { on } \partial \Sigma .
\end{aligned}
$$

Proof Let $\stackrel{\circ}{H}_{1}^{2}$ be the Hilbert completion of the space $C_{0}^{\infty}(\stackrel{\circ}{\Sigma})$ of smooth functions

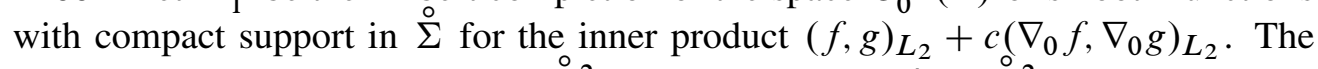
Riesz representation theorem in $\stackrel{\circ}{H}_{1}^{2}$ yields a solution $\widehat{w} \in \stackrel{\circ}{H}_{1}^{2}$ for the equation $\Delta_{0} \widehat{w}=-c \hat{w}+\hat{\psi}$, where $\hat{\psi}:=\psi-c v_{+}-\Delta_{0} v_{+}$lies in $C^{\infty}(\Sigma)$. Standard elliptic regularity and Sobolev embedding (see eg Gilbarg and Trudinger [7, 8.13 and 7.26]) ensure that $\widehat{w} \in C^{\infty}(\Sigma)$. The function $w:=\widehat{w}+v_{+}$is the desired solution.

Corollary 6.7 Let $\Sigma$ be a compact surface with smooth boundary, $f \in C^{\infty}(\mathbb{R} \times \Sigma)$ and $c>0$ be a constant such that (6-4) holds. Let $\psi, v_{+}$and $v_{-}$be functions in $C^{\infty}(\Sigma)$, and assume that $v_{-} \leq v_{+}$, and that $\Delta_{0} v_{+} \geq f\left(x, v_{+}\right)$holds while $\Delta_{0}\left(x, v_{-}\right) \leq f\left(x, v_{-}\right)$. Then, the nonlinear Dirichlet problem

$$
\begin{aligned}
\Delta_{0} v & =f(x, v) \quad \text { on } \stackrel{\circ}{\Sigma}, \\
v & =v_{+} \quad \text { on } \partial \Sigma,
\end{aligned}
$$

admits a solution $v \in C^{\infty}(\Sigma)$ with $v_{-} \leq v \leq v_{+}$. 
Proof We use an iterative scheme. Begin with $w_{0} \equiv v_{+}$, and let $w_{j} \in C^{\infty}(\Sigma)(j \geq 1)$ be the solution of the linear Dirichlet problem

$$
\begin{aligned}
\Delta_{0} w_{j} & =-c w_{j}+f\left(x, w_{j-1}\right)+c w_{j-1} \quad \text { on } \stackrel{\circ}{\Sigma}, \\
w_{j} & =v_{+} \quad \text { on } \partial \Sigma,
\end{aligned}
$$

provided by Lemma 6.6. The maximum principle and property (6-4) ensure recursively that $\left(w_{j}\right)$ is a decreasing sequence, that the $w_{j}$ are upper solutions for Equation (6-5), and that $v_{-} \leq w_{j} \leq v_{+}$holds for $j \geq 1$. Global elliptic estimates and Kondrakov embedding (Gilbarg and Trudinger [7, 9.14 and 7.26]) show that the sequence $w_{j}$ is bounded in $C^{1, \alpha}(0<\alpha<1)$. Hence a subsequence of $\left(w_{j}\right)$ converges uniformly on $\Sigma$ to a weak solution $v$ of Equation (6-5). Elliptic regularity ensures that $v$ is smooth.

End of proof of Proposition 6.5 For each compact surface $\Sigma_{k} \subset S$, let $v_{k} \in C^{\infty}\left(\Sigma_{k}\right)$ with $v_{-} \leq v_{k} \leq v_{+}$be the solution of the Dirichlet problem (6-5) on $\Sigma_{k}$ given by Corollary 6.7. The maximum principle ensures that the sequence $\left(v_{k}\right)$ is decreasing, namely that $v_{k+1} \leq v_{k}$ holds on $\Sigma_{k}$. Since the sequence is bounded below, it converges to a weak bounded solution $v$ of Wang's equation. Elliptic regularity again shows that $v$ is smooth.

Uniqueness of a bounded solution for Equation (6-2) is an immediate consequence of the generalized maximum principle of Yau (Theorem 5.3).

When the surface $S$ is compact, a bounded solution is provided by the same recursive scheme-without boundary conditions - and uniqueness of a bounded solution follows from the classical maximum principle.

Remark 6.8 Denote by $\mathcal{G}$ the set of properly convex projective structures with finite volume on $S$, quotiented by the natural action of the group $\operatorname{Diff}_{0}(S)$ of diffeomorphisms isotopic to the identity map (see Goldman [9]). One of the interesting features of Theorem 1.1 is that, by applying Riemann-Roch, it allows us to identify $\mathcal{G}$ with $\mathbb{C}^{8(g-1)+3 r}$, where $g$ is the genus of the compactification $\bar{S}$ of $S$, and $r$ is the number of punctures. In particular, it endows $\mathcal{G}$ with a canonical complex structure.

\section{References}

[1] L V Ahlfors, An extension of Schwarz's lemma, Trans. Amer. Math. Soc. 43 (1938) 359-364 MR1501949

[2] J-P Benzécri, Sur les variétés localement affines et localement projectives, Bull. Soc. Math. France 88 (1960) 229-332 MR0124005 
[3] E Calabi, Complete affine hyperspheres, I, from: "Symposia Mathematica, Vol. X", Academic Press, London (1972) 19-38 MR0365607

[4] SY Cheng, ST Yau, On the regularity of the Monge-Ampère equation $\operatorname{det}\left(\partial^{2} u / \partial x_{i} \partial x_{j}\right)=F(x, u)$, Comm. Pure Appl. Math. 30 (1977) 41-68 MR0437805

[5] S Y Cheng, S T Yau, Complete affine hypersurfaces, I, The completeness of affine metrics, Comm. Pure Appl. Math. 39 (1986) 839-866 MR859275

[6] S Gigena, On a conjecture by E. Calabi, Geom. Dedicata 11 (1981) 387-396 MR637915

[7] D Gilbarg, NS Trudinger, Elliptic partial differential equations of second order, Classics in Mathematics, Springer, Berlin (2001) MR1814364

[8] W Goldman, Projective geometry on manifolds, Lecture notes (1988) Available at http : //www2. math. umd. edu/ wmg/pgom.pdf

[9] W M Goldman, Convex real projective structures on compact surfaces, J. Differential Geom. 31 (1990) 791-845 MR1053346

[10] F Labourie, Flat projective structures on surfaces and cubic holomorphic differentials, Pure Appl. Math. Q. 3 (2007) 1057-1099 MR2402597

[11] J Lee, Convex fundamental domains for properly convex real projective structures Available at http://users.math.yale.edu/ j1232/

[12] A M Li, Calabi conjecture on hyperbolic affine hyperspheres, II, Math. Ann. 293 (1992) 485-493 MR1170522

[13] J Loftin, Applications of affine differential geometry to $\mathbb{R}^{2}$ surfaces, $\mathrm{PhD}$ thesis, Rutgers University (1999) Available at http://andromeda.rutgers.edu/ loftin

[14] J C Loftin, Affine spheres and convex $\mathbb{R P}^{n}$-manifolds, Amer. J. Math. 123 (2001) 255-274 MR1828223

[15] J C Loftin, The compactification of the moduli space of convex $\mathbb{R P}^{2}$ surfaces, I, J. Differential Geom. 68 (2004) 223-276 MR2144248

[16] J Loftin, Survey on affine spheres, from: "Handbook of geometric analysis, No. 2", (L Ji, P Li, R Schoen, L Simon, editors), Adv. Lect. Math. (ALM) 13, Int. Press, Somerville, MA (2010) 161-191 MR2743442

[17] L Marquis, Espace des modules marqués des surfaces projectives convexes de volume fini, Geom. Topol. 14 (2010) 2103-2149 MR2740643

[18] L Marquis, Surface projective convexe de volume fini, Ann. Inst. Fourier (Grenoble) 62 (2012) 325-392 MR2986273

[19] K Nomizu, T Sasaki, Affine differential geometry, Cambridge Tracts in Mathematics 111, Cambridge Univ. Press (1994) MR1311248

[20] M Troyanov, The Schwarz lemma for nonpositively curved Riemannian surfaces, Manuscripta Math. 72 (1991) 251-256 MR1118545 
[21] NS Trudinger, X-J Wang, The Monge-Ampère equation and its geometric applications, from: "Handbook of geometric analysis, No. 1", (L Ji, P Li, R Schoen, L Simon, editors), Adv. Lect. Math. (ALM) 7, Int. Press, Somerville, MA (2008) 467-524 MR2483373

[22] CP Wang, Some examples of complete hyperbolic affine 2-spheres in $\mathbb{R}^{3}$, from: "Global differential geometry and global analysis", (D Ferus, U Pinkall, U Simon, B Wegner, editors), Lecture Notes in Math. 1481, Springer, Berlin (1991) 271-280 MR1178538

[23] S T Yau, Harmonic functions on complete Riemannian manifolds, Comm. Pure Appl. Math. 28 (1975) 201-228 MR0431040

Département de Mathématiques, Université Paris-Sud Bâtiment 425, Faculté des Sciences d'Orsay, 91405 Orsay Cedex, France yves.benoist@math.u-psud.fr, dominique.hulin@math.u-psud.fr http: //www . math.u-psud.fr/ benoist

Proposed: Danny Calegari

Seconded: Jean-Pierre Otal, Benson Farb
Received: 12 May 2012 Accepted: 10 November 2012 